Communication, technologies et développement

5 | 2018

Le numérique et le développement des Suds

\title{
Analyse d'anciennes et nouvelles idéologies du développement en Afrique
}

Jean-Paul Lafrance

\section{OpenEdition}

Journals

Édition électronique

URL : http://journals.openedition.org/ctd/285

DOI : $10.4000 /$ ctd. 285

ISSN : 2491-1437

Éditeur

Chaire Unesco Pratiques émergentes en technologies et communication pour le développement

Référence électronique

Jean-Paul Lafrance, « Analyse d'anciennes et nouvelles idéologies du développement en Afrique », Communication, technologies et développement [En ligne], 5 | 2018, mis en ligne le 02 janvier 2018, consulté le 02 mai 2019. URL : http://journals.openedition.org/ctd/285 ; DOI : 10.4000/ctd.285

Ce document a été généré automatiquement le 2 mai 2019.

Communication, technologies et développement 


\title{
Analyse d'anciennes et nouvelles idéologies du développement en Afrique
}

\author{
Jean-Paul Lafrance
}

\section{NOTE DE L'AUTEUR}

\section{SWAHILI}

Muhtasari: Gilbert Rist anaeleza kama "maendeleo" ni utopia ya inchi za Magharibi na kipengele cha dini ya kisasa ya maendeleo ya ustaarabu. Je! Dhana hii inaweza kutumika kama vile Afrika? Mwandishi anachungulia kuzaliwa kwa dhana ile mu kisiasa, kama dhana ya maendeleo asema "développementiste", tarehe kutoka mwishoni mwa Vita Kuu ya Pili ya Dunia, wakati ambapo redefinition ya kisiasa ya kigeni ya Marekani. Lakini kuwaza maendeleo, ni kuwaza maendeleo duni pia, ao kusema na heshima inchi zinazoendelea, na kila kitu kinachowashawishi kwa hali ya walewa inchi. Ata vile, Africa inaweza kumudu maendeleo ya teknolojia? Swali kubwanikujiulizaakama Afrika inahitaji maendeleo gani ? Tatmini ya vipande mazuri na vipenpande baya ya maendeleo ya nchi za Afrika, na fursa za kufanikiwa.

1 Il s'agit de présenter et d'analyser les paradigmes de l'aide au développement en Afrique, soit celui du modernisme dit développementiste proposé à la Société des Nations par les États-Unis à la fin de la Seconde Guerre mondiale, et celui dit de la société numérique au début du $\mathrm{XX}^{\mathrm{e}}$ siècle. Nous donnerons quelques éléments d'une critique de ces manières de penser et de faire. Nous montrerons enfin, en conclusion, pourquoi il est nécessaire que les Africains se forgent eux-mêmes leur propre modèle de développement, en tenant compte de leurs forces et faiblesses, au moment où l'ensemble du monde a intérêt à les associer au règlement des problèmes généraux du monde, soit l'exode migratoire, l'épidémie mortifère du terrorisme de l'État islamique (DAESH) et les changements climatiques. 


\section{Le paradigme développementiste (1949)}

\section{Histoire d'une croyance occidentale}

2 Gilbert Rist ${ }^{1}$ considère le développement comme une utopie et un élément de la religion moderne du progrès des civilisations. Sans remonter jusqu'à la notion aristotélicienne de la « nature des choses » - que l'on peut assimiler au développement - ou à la théologie augustinienne de l'histoire du salut, c'est au XVII e siècle que Descartes établit la prééminence de la Raison et la nécessité du progrès de la connaissance ; au siècle suivant, pendant la période des Lumières, personne ne doute du principe de la croissance quasi infinie des civilisations. Hormis le pessimisme de Rousseau qui croit que c'est la société qui corrompt l'homme naturellement bon, l'idéologie du progrès triomphe partout dans la philosophie allemande, de Kant à Hegel et à Marx, quoique l'on ne s'entende pas sur la nature du mal. «Ainsi au cœur du dispositif occidental se trouve l'idée qu'il existe une histoire naturelle de l'humanité, c'est-à-dire que le développement des sociétés, des connaissances et de la richesse correspond à un principe naturel, autodynamique, qui fonde la possibilité d'un grand récit ${ }^{2} »$. L'évolutionnisme social s'appuie sur la croyance en la croissance exponentielle de la richesse des nations, grâce à l'industrialisation, aux résultats du développement scientifique et à la mise au point des techniques modernes. Cette théorie de l'histoire humaine, bien loin de la pensée orientale par exemple, est probablement la conséquence d'une laïcisation de la religion chrétienne.

\section{Naissance politique du paradigme}

3 À la fin de la Seconde Guerre mondiale, la politique extérieure des États-Unis est en pleine redéfinition ${ }^{3}$. Ces derniers sont devenus une super nation et sont convaincus qu'ils ont un grand rôle à jouer dans le monde. Les pays européens par contre ont beaucoup souffert de la guerre et peinent à sortir du colonialisme. Le 20 janvier 1949, le président Truman dans son discours traditionnel sur l'État de l'Union sort de son chapeau, à la façon d'un magicien, une formule qui deviendra la charte du développement :

«Il nous faut lancer un nouveau programme qui soit audacieux et qui mette les avantages de notre avance scientifique et de notre pensée industrielle au service de l'amélioration et de la croissance des régions sous- développées. Plus de la moitié des gens de ce monde vivent dans des conditions voisines de la misère... Les ÉtatsUnis occupent parmi les nations une place prééminente quant au développement des techniques industrielles et scientifiques " ${ }^{4}$.

4 Le binôme pays développés/pays sous-développés vient d'être inventé de toutes pièces et remplace la division ancienne entre pays colonisateurs et pays colonisés. Dès le 16 novembre 1949, l'Assemblée générale des Nations Unies approuve la création du programme élargi d'assistance technique et plus tard, le transfert de capitaux du Nord vers le Sud via la Banque Mondiale.

5 Ce n'est qu'au XXe siècle que les États ont cru pouvoir diriger le développement d'une façon significative, en se donnant des objectifs précis en fonction des chiffres de la comptabilité nationale (augmentation du PIB ou de la richesse des familles, développement du commerce extérieur, accroissement de la durée de vie et diminution de la mortalité infantile, croissance du taux d'alphabétisation, etc.). 
6 Toutefois, ce n'est que plus tard que certains pays (dits riches) se sont mêlés de vouloir «aider au développement » d'autres pays plus pauvres; par souci de commisération, par charité chrétienne, par grandeur d'âme, on peut en douter! L'instinct de puissance, le désir de conquête et l'appétit de richesses ont toujours été plus forts que la pitié, du moins pour ce qui concerne les nations... Mais les grandes guerres du $\mathrm{XX}^{\mathrm{e}}$ siècle avaient laissé certaines populations dans un tel état de désolation (pensons à l'Europe, à l'Allemagne et au Japon) après la Seconde Guerre que les États-Unis et les autres pays industrialisés ont eu peur de créer des déséquilibres profonds dans l'état du monde ${ }^{5}$. On a compris que les guerres engendrent les guerres, que la richesse crée la richesse, qu'un état extrême de pauvreté génère la violence, le terrorisme et les flux migratoires vers les pays riches. De nos jours, ajoutons que les problèmes de la crise écologique et du climat provoquent ce même sentiment d'urgence.

7 Après 1945, les pays vainqueurs abordèrent, dans les grandes conférences internationales, «la question du développement». Actuellement, au sein de l'ONU, de l'Union européenne, aux États-Unis et dans les autres grands pays du monde, on a l'impression que l'humanité tout entière se sent à nouveau déstabilisée et exige des solutions globales à des problèmes mondiaux; les nations se réunissent autour de la COP21 (Paris) pour tenter de trouver une solution aux changements climatiques; par ailleurs, la crise européenne des migrants et les actions terroristes de l'État islamique (DAESH) et d'AlQaïda dans les pays africains, au Moyen-Orient et dans la majorité des États du monde forcent les pays industrialisés à trouver une solution globale.

\section{Les travers de l'aide au développement}

Qui dit pays développé, dit pays sous-développé, ou pour être plus poli pays en voie de développement ${ }^{6}$. Les États-Unis, grands vainqueurs de la Seconde Guerre mondiale, sont les premiers à établir une problématique d'aide au développement et à dégager dans leur comptabilité nationale des budgets conséquents. De nos jours, on fixe à $0,7 \%$ du PIB le taux de l'aide acceptable, ce que la grande majorité des pays n'arrive plus à atteindre. En conséquence, depuis 50 ans, des masses considérables de ressources financières sont disponibles pour permettre aux pays pauvres de « rattraper » les pays riches.

Le paradigme développementiste, puisqu'il faut l'appeler par son nom, domine les théories du développement jusqu'aux années 1970 et au-delà. Ce dernier se définit comme un processus de rattrapage par lequel les pays du Sud doivent se moderniser en prenant les pays du Nord comme modèle ; il consiste en un accroissement de la productivité des nations pauvres qui, pour ce faire, doivent intégrer les connaissances et adopter les technologies du Nord. Walt Whitman Rostow considérait que la société traditionnelle était le degré zéro de l'histoire ${ }^{7}$ et décrivait l'industrialisation comme un processus unilinéaire et irréversible qui ne pouvait suivre qu'un seul chemin dans le processus d'industrialisation: celui tracé par les pays du Nord, dont l'exemple fut au XIX siècle, l'Angleterre suivie par la France, les États-Unis, l'Allemagne et les autres pays de l'Europe, et, plus tard, le Japon...

10 Évidemment, la situation a bien changé après les années 70 à la suite de la création des pétro-États (les pays du Golfe, l'Algérie, l'Indonésie, l'Iran, le Nigeria et le Venezuela), conséquence du choc pétrolier et de l'action de l'OPEP ; 20 ans plus tard naissait ce que l'on a appelé le groupe des cinq pays émergents, BRICS : Brésil, Russie, Inde, Chine et Afrique du Sud. Malgré l'apport en capital dû à l'exploitation insensée de leurs ressources 
non renouvelables, plusieurs de ces pays n'ont pas réussi à régler leurs problèmes de pauvreté et d'égalisation des chances pour leurs citoyens ${ }^{8}$. Comme on peut le constater, ce n'est pas l'aide au développement qui est la cause du « décollage » de ces pays devenus rapidement très riches. Il existe d'autres raisons qui expliquent le développement remarquable de certains, comme la Corée du Sud ou les pays nordiques.

\section{La nouvelle idéologie du développement de l'Afrique : de la nécessité de profiter de la révolution communicationnelle et numérique de l'Occident}

11 À la fin du XXe siècle, l'Afrique se trouve confrontée à un nouveau paradigme. À l'époque, le continent africain ne peut communiquer avec le reste du monde qu'en passant par les réseaux du Nord. Son réseau téléphonique est désuet et mal entretenu. Par exemple, en 2000, le continent comptait seize millions de raccordements téléphoniques, un chiffre inférieur au nombre de lignes que l'on trouvait à Manhattan ou à Tokyo. Peu d'utilisateurs avaient accès à Internet. Sans jeu de mots, l'Afrique était un vaste «trou noir » qui ne profitait pas ou peu du mouvement général de la mondialisation. La grandmesse qui célèbre l'arrivée du pan communicationnisme, c'est le SMSI (le Sommet mondial sur la société d'information) tenu à Genève en 2003 par l'UIT (Union International des Télécommunications), qui annonce l'ère nouvelle d'internet, du téléphone mobile et des réseaux sociaux. "OrdinateurR4s, téléphones et smartphones en main, des citoyens testent, imaginent, contournent les lois, sans vouloir être forcément dans l'illégalité, pour communiquer, acheter, s'exprimer, apprendre différemment. Il ne s'agit pas d'une révolution numérique, mais d'une civilisation numérique ${ }^{9}$ ", estime Irina Bokova, directrice de l'UNESCO, qui résume bien l'idéologie véhiculée par les promoteurs des NTIC (Nouvelles techniques d'information et de communication).

En fait, bien avant l'an 2000, l'Afrique avait déjà commencé à profiter des largesses des organismes internationaux (Fonds Monétaire International, Banque mondiale, Francophonie) et de plusieurs pays occidentaux (Québec, France, États-Unis) pour l'installation de médias communautaires (radios locales, télévisions éducatives, centres informatiques, universités à distance, etc.). On parlait à l'époque de communication du développement, ou mieux de "communication pour le développement» ou de « communication pour le changement social». Dans un colloque tenu à l'UQAM et relaté par Agbobli et Aubin :

«Alzouma se penche sur la reformulation du discours développementaliste élaboré autour des TIC et qui tend de plus en plus à abandonner toute référence aux ordinateurs pour se concentrer sur le téléphone mobile, ses usages et ses appropriations. Il repère trois modes d'appropriation innovants: les modes d'appropriation et d'usages nouveaux qui consistent essentiellement, pour les utilisateurs africains, à s'adapter dans un contexte de ressources limitées en capital économique et culturel ; l'adaptation des objets techniques eux- mêmes (préconisée en Inde) ; et la convergence numérique. Il démontre que ces modes d'appropriation dits innovants manifestent en fait des disparités en termes d'accès, mais aussi une inégale distribution des compétences techniques et culturelles (éducation) ${ }^{10}$ ».

Il indique bien une autre façon de voir le développement au temps du numérique, alors que l'on ne parle que des problèmes techniques autour de la fracture numérique. 

numérique en Afrique empruntent le plus souvent une approche essentiellement technodéterministe. Missé Missé, dans le même colloque :

«insiste sur sa fonction idéologique et instrumentale [qui] envisage la communication pour le changement social comme un ensemble institutionnalisé de pratiques de médiation, qui manquent généralement de pertinence sociale et qui mobilisent des méthodes et des technologies comme des mécanismes de légitimation du pouvoir des pays occidentaux et de leurs agences de développement ${ }^{11} »$.

\section{L'Afrique doit-elle bouder le développement technologique?}

Pourquoi l'Afrique en général a-t-elle été tenue à l'écart de la modernité ? Malédiction des pays du Sud? Atavisme? Non, d'autres pays de l'hémisphère sud comme la Chine, la Corée du Sud, en partie l'Inde et plusieurs pays du Sud-Est asiatique sont en train de sortir du sous-développement. L'Afrique est-elle réfractaire à l'idée de progrès? La culture, la mentalité africaine, les traditions sont-elles imperméables aux idées nouvelles? Ce continent qui a vu naître l'Homo sapiens est-il étranger aux vertus de la technologie nouvelle ? L'Afrique est-elle forcée d'être à la traîne en raison de son passé trop lourd? Mais l'Afrique est riche de ses jeunes, de ses populations, de ses ressources naturelles, de ses traditions culturelles... Les nouvelles technologies intelligentes sont bénéfiques pour l'humanité, mais ses bienfaits doivent être distribués à tous et non pas servir à accumuler les centaines de milliards d'euros du $10 \%$ des entreprises multinationales occidentales et de leurs dirigeants ${ }^{12}$.

Pour Axelle $\mathrm{Kabou}^{13}$, les causes de l'inertie de l'Afrique en ce qui a trait au modernisme sont les suivantes :

manque de capitaux. Selon Le CRDI (centre de recherche en développement international du Canada), 25 à $40 \%$ de l'aide au développement disparaît dans les goussets des élites. Jacques T. Godbout prétendait qu'il faudrait refuser toute l'aide, parce qu'elle crée un état de dépendance ${ }^{14}$. L'Afrique aurait intérêt à utiliser plutôt les taxes et les impôts pour se financer et à utiliser davantage le microfinancement, le sociofinancement et le financement de proximité pour ses projets locaux (qui est la véritable économie du partage collectif). Le concept de ce que les Québécois appellent l'économie sociale ${ }^{15}$ doit être analysé comme un remède au financement des petites économies. La stagnation de l'Afrique est-elle toujours la faute des autres? Certes, l'Afrique a été victime de la traite négrière, de la colonisation des pays du Nord, de l'apartheid, des politiques économiques du FMI et de la Banque mondiale, de la déterritorisation des échanges, du pillage de ses ressources naturelles, etc., mais elle devra sortir de ce complexe de victimisation pour prendre en mains son propre développement.

L'exode d'Africains qui ont été formés dans les meilleures universités, mais qui ne reviennent pas chez eux pour faire évoluer leur pays. D'où la nécessité du rapatriement des cerveaux. Encore faudrait-il leur donner du travail et une place au soleil, disent certains!

19 L'existence d'une multitude de roitelets prévaricateurs qui ont pris le contrôle de leur pays après son indépendance, protégés en cela par les anciens colonisateurs. La tendance à dire que la démocratie n'est pas dans les gênes des Africains ou que la démocratie ne 
respecte pas la culture ancestrale africaine conforte les oligarchies qui protègent leur clan, leur famille et leur compte en banque. Comme les Occidentaux, l'Afrique a aussi son $1 \%$ d'élite prévaricatrice.

La grande question est de se demander de quel type de développement l'Afrique a besoin. Contrairement à ce que pensaient Walt $\mathrm{W}$. Rostow et les chantres de la thèse développementiste des années 1960, aucun pays n'est obligé d'adopter le modèle libéraloconsumériste américain. Il existe maintenant d'autres façons de se moderniser ; on parle $\mathrm{du}$ modèle écologiste, misant sur le développement responsable et les ressources renouvelables, de celui de la société numérique, de celui du socialisme. Par exemple, il est clair que le développement de la Chine, s'appuyant sur un gouvernement fort et une organisation collective en phase avec leur éthos et leurs traditions séculaires qui privilégient la collectivité plutôt que l'individu, est fort différent de celui des États-Unis. Plusieurs Africains formés dans les universités américaines ou européennes rêvent tout simplement de copier les réalisations du Nord. Or, si l'Afrique a fait d'énormes progrès pour développer son système de communication (en 2015, on comptait 700 millions d'abonnés au téléphone mobile), c'est qu'elle a adopté un modèle communautaire; elle a fait le pari de ne pas passer par le téléphone filaire et d'installer un réseau mobile. De plus, le combiné mobile est davantage le fait d'une utilisation collective que privative, le fameux modèle du callbox qui permet à 7 personnes sur 10 d'avoir l'accès au mobile ${ }^{16}$ et au mobile banking. Les secteurs de la santé et de la médecine, de l'enseignement supérieur, de la recherche scientifique auraient intérêt à explorer en Afrique l'avantage des liaisons numériques pour asseoir ses institutions et développer ses réseaux commerciaux avec le nord.

21 Pour Thomas Sankara, le leader charismatique assassiné en 1987, l'Afrique doit refuser l'assistance qui « développe la mentalité d'assisté ${ }^{17}$ ", comme l'a fait dernièrement l'Inde ; il fut peut-être le premier à le dire, mais il est loin d'être le seul à le prétendre, car l'aide au développement crée la dépendance, en plus d'encourager la corruption des élites et de conforter le népotisme.

\section{Une nouvelle idéologie}

La révolution numérique est-elle un gage de salut pour le réveil de l'Afrique ? Oui et non, car le changement ne peut s'installer que si certaines conditions sociopolitiques et économiques le rendent possible. L'humanisme numérique est incompatible avec le néolibéralisme de type prévaricateur. En ce qui concerne la révolution numérique que certains appellent la quatrième révolution industrielle, on nage en pleine euphorie ou on pose les pires réserves.

\section{Des effets positifs}

Évidemment, l'arrivée des technologies intelligentes, de l'internet des objets et des mégadonnées (big data) a des effets positifs: une communication plus efficace, des services financiers plus faciles d'accès, des activités commerciales moins coûteuses et un grand potentiel d'innovation dans la livraison des services et la fabrication des objets de la société moderne. La médecine a fait des bonds considérables, la longévité de la vie humaine est en pleine croissance, l'automobile est plus sécuritaire et moins polluante, l'information est plus facilement disponible, etc. Le nombre d'internautes est passé d'un milliard en 2005 à 3,5 milliards en 2016, ce qui fait qu'un habitant sur deux du monde est 
branché. Cependant, le grand paradoxe pour les $20 \%$ les plus pauvres, c'est qu'il y a plus de personnes qui ont accès à la téléphonie cellulaire qu'à l'eau salubre, à l'électricité et aux installations d'hygiène comme les toilettes ${ }^{18} .$.

\section{Et des ratés...} Schwab, son directeur, et reconnaître que « la quatrième révolution industrielle risque d'alimenter les tensions sociales, d'accentuer les inégalités, de polariser davantage la classe moyenne et d'exacerber les perturbations sur le marché du travail, accentuant le clivage entre les emplois qualifiés bien rémunérés et ceux peu ou pas qualifiés et faiblement rémunérés (...). Cette quatrième révolution industrielle invoquée, celle du numérique, renferme déjà dans ses gènes des ratés de la dernière vague : les effets de la technologie sur la productivité mondiale et l'amélioration des opportunités pour les pauvres et la classe moyenne qui n'a pas été à la hauteur des attentes ${ }^{19}$ ». En un mot, un petit nombre d'individus et d'entreprises se sont accaparé les profits de la révolution numérique, aux dépens de la classe moyenne et des démunis. Les nouvelles technologies bouleversent le monde du travail, mais ne profitent pas à la grande majorité des citoyens de la classe moyenne.

Il existe donc une incompatibilité entre le rêve élitiste de certains prospectivistes et la possibilité de faire profiter à tous de l'amélioration de leurs conditions de vie grâce aux nouvelles technologies. L'humanisme numérique poussé dans ces dernières élucubrations s'appuie sur une philosophie néolibérale qui favorise uniquement une minorité d'individus capables de profiter des progrès des NTIC et des NBIC ${ }^{20}$. Ainsi, l'humanisme numérique adopte une approche abstraite et théorique sur les bénéfices des techniques émergentes en santé. Qui aura les moyens de se procurer les prothèses nécessaires pour remplacer les organes déficients? Qui pourra se payer les composants chimiques qui prolongent la vie? Le numérique crée une minorité d'emplois à haute technicité, mais déclasse un grand nombre d'emplois dits non spécialisés qui ne permettent plus à des travailleurs de vivre décemment. Pour remédier à ces problèmes, nos sociétés ont besoin de remettre en cause toute leur philosophie du travail et de l'emploi. L'économiste John Mitchell ${ }^{21}$ se demande s'il ne faut pas financiariser la technologie, comme on a dû le faire pour soutenir l'environnement en créant de toutes pièces le développement durable et en créant un impôt sur les richesses non renouvelables! Les États doivent mettre sur pied une économie verte pour éviter la catastrophe environnementale : est-ce nécessaire de songer à une "taxe rose" (pour Robots, Ordinateurs et Systèmes experts), comme le suggère John Mitchell ${ }^{22}$, pour éviter la paupérisation d'une partie de plus en plus importante de la population du monde. Il existe une véritable rupture entre les élites économiques et les travailleurs à faible revenu. Un humanisme qui n'est pas redistributeur des profits des Nouvelles Technologies Intelligentes (NTI) n'est pas synonyme de progrès pour tous.

Il faut rapidement transformer l'économie actuelle (de type néolibéral) en économie intelligente, basée sur la Recherche et Développement, qui crée des surplus qui doivent être redistribués à tous, pour le bien-être de la société. La civilisation numérique doit bouleverser notre philosophie du travail et de l'emploi et non pas servir à enrichir les plus riches. 


\section{Le déficit numérique} stylo- bille, comme au temps des luddites (en 1811-1812 en Angleterre) qui brisaient les métiers à tisser pour sauvegarder leurs emplois. On ne peut nier le phénomène non plus, comme le font les gouvernements ou les aficionados des NTIC, ou croire qu'en mettant l'accent sur la Recherche et Développement ou la délocalisation des entreprises, on règlera le problème. Et ce qui encore pire à l'heure actuelle, c'est que les profits de la suppression d'emplois permettent la croissance des portefeuilles des PDG et des hauts gradés des entreprises.

31 Certains proposent carrément de hausser les taxes sur l'usage des technologies, comme on a fini par accepter le principe de l'utilisateur-payeur en matière d'environnement, ce qui ne fut pas une mince affaire et qui est encore peu appliqué en ce qui concerne les ressources naturelles non renouvelables. Une telle mesure, encore impensable, permettrait de prévoir un revenu minimum garanti ${ }^{26}$ pour les chômeurs de longue durée, mais c'est difficilement envisageable, car elle créerait, dit-on, une société de sans-emplois à demeure...

- la suppression importante d'emplois due à la numérisation du travail,

- l'évasion fiscale, conséquence de la virtualisation de l'argent et de la financiarisation de l'économie,

- la création de super entreprises, telles les GAFA (Google, Apple, Facebook et Amazon), qui sont en train de monnayer le Web à leur profit.

\section{L'automatisation des tâches routinières}

Elle supprime de plus en plus d'emplois en Amérique, en Europe et en Asie. Et quand nous disons « routinières », il faut savoir qu'étant donné le progrès de l'intelligence artificielle et de la robotique, les machines intelligentes peuvent de plus en plus remplacer les humains. L'économie, quoique ralentie, continue de croître, mais le chômage aussi, quelles que soient les mesures de soutien à l'emploi que proposent les gouvernements et qui sont de plus en plus inefficaces (le cas est pathétique en Europe, notamment en France). Il se crée des emplois de haut niveau certes, mais aussi des emplois à bas salaires (restauration, commerce de détail, gardiennage, services à la personne, etc.) et on délocalise le travail dans les pays moins chers ${ }^{23}$; le déficit est manifeste. Tous les jours, on supprime des emplois : la fonction publique de tous les pays, l'armée, la banque, etc. On prétend qu'un million d'emplois disparaîtront aux États-Unis et un autre en Australie dans les prochaines années. Maintenant, la saignée rattrape le secteur des NTIC : Intel en abolit 12 000, Microsoft en fait disparaitre 1800 depuis l'intégration de Nokia qui en avait finissants ne trouvent rien à leur sortie du collège ou bien, acceptent des boulots qui ne nécessitent aucun diplôme d'études supérieures ${ }^{25}$. »

\footnotetext{
(a)
} 

révolution industrielle, passant de 10 et 12 heures par jour à 9, 8, 7, 6 heures, pendant que la production augmentait du même coup ; pendant la révolution numérique, les heures de labeur devraient continuer à diminuer passant à 5, 4, 3 heures par jour, de 35 heures/ semaine à 32, 30, 25 heures, pendant que les activités culturelles, agricoles, communautaires et de santé ne cesseront de croître chez les gens moins accaparés par le travail rémunéré. Bien que les expériences faites dans les pays scandinaves sur la réduction du temps de travail montrent que la productivité augmente d'une façon importante et dans le secteur public et dans le secteur privé, l'industrie n'y croit pas encore ${ }^{27}$. C'est donc toute la philosophie du travail rémunéré et non rémunéré qu'il faut changer, d'où la nécessité d'une révolution des mentalités des citoyens, des politiques et des chefs d'entreprises.

Évidemment la controverse fait rage chez les scientifiques entre ceux qui arguent que le numérique crée énormément d'emplois de haute qualitée ${ }^{8}$ et ceux qui prétendent que les travailleurs sont en train de perdre la course contre les automates ${ }^{29}$. Comme toute controverse, chacun avance des arguments sur ses prévisions dans un domaine fort complexe où les solutions ne sont pas faciles; nous avons tous connu la période où l'on s'imaginait que tout ce qui sortait du numérique était gratuit... Il est urgent de financiariser les technologies. Les réflexions des pays industrialisés sur les effets négatifs de la révolution numérique sur l'emploi ne s'appliquent peut-être pas telles quelles en Afrique, car celle-ci profite en ce moment de la mondialisation et de la délocalisation des entreprises; ainsi la Chine commence à délocaliser son industrie manufacturière en Éthiopie en raison des bas salaires. Mais les effets ne tarderont pas à se faire sentir sur le continent africain, d'autant plus que la majorité des emplois sont de « petits boulots » et qu'une grande partie de l'économie de ses pays fonctionne encore sur le modèle traditionnel.

\section{La nature virtuelle du numérique}

Autre controverse qui montre que le numérique, en raison de sa nature virtuelle ${ }^{30}$, a permis d'éviter l'impôt aux individus et aux sociétés et de déjouer les lois et les règlementations des États qui sont toujours quelques années en retard sur les inventeurs, les entreprises et les consommateurs. On l'a dit et redit, les données numériques voyagent et franchissent les frontières incognito et ne s'arrêtent pas au point de contrôle pour payer leurs taxes et leurs impôts. En plus, les entreprises, les courtiers, les PDG et les investisseurs savent comment faire ce qu'ils appellent la "comptabilité créative", souvent en toute légalité, pour déplacer leurs profits dans les îles paradisiaques qui proposent d'éviter la taxation. Livres, films, logiciels, services voyagent à l'air libre. Qui faut-il taxer pour rétablir la justice sociale? Les entreprises de distribution électronique, les matériels de visionnement, les clients, mais comment? Dans l'économie dite de partage du type AIRBNB (fondée par Chesky et Gebbia à San Francisco en octobre 2007), Uber (fondée par T. Kalanick et valorisée à 50 milliards de dollars en 2015), Lyft (encore situé à San Francisco), les grands du commerce électronique (Amazon, Alibaba et autres) et les réseaux de financement participatif (crowdsourcing) comme Kickstarter (fondé en 2009 par des investisseurs de la Silicon Valley), on peut à peu près tout produire, distribuer et utiliser les services de transport, d'habitation, de restauration, de soins personnels, de loisirs et de communication, à ce point que pour certains, il faudrait plutôt parler de la 
révolution économique $2.0^{31}$. Il faut cependant rappeler que toutes ces entreprises dites collaboratives (toutes d'origine américaine ou du moins en provenance des pays industrialisés), gardent $30 \%$ de la trésorerie pour leurs services généraux et distribuent à leurs nombreux collaborateurs quelques miettes en retour, de quoi améliorer leurs fins de mois...

\section{La création de super entreprises}

Ces super-entreprises, telles les GAFA, sont en train de monnayer le Web à leur profit. Internet a été créé par un consortium d'entreprises publiques et de chercheurs universitaires, qui avaient inscrit dans leurs pratiques une philosophie de service public ( communs ${ }^{32}$ ). À l'origine les initiateurs avaient établi la neutralité du Réseau des réseaux et une philosophie du partage entre les usagers, tour à tour producteurs d'informations et utilisateurs du trésor que constitue le grand réseau ${ }^{33}$. Or, voici que de grandes entreprises médiatiques produisent des systèmes-propriétaires et engrangent d'énormes fortunes, offrent des services faussement gratuits, puisqu'elles exigent des usagers qu'ils abandonnent tous leurs droits. Facebook fait toute une série d'acquisitions (Instagram, WhatsApp, Oculus VR), Microsoft s'est emparée de Nokia, Skype, Linked in et Hotmail, Google s'est acheté YouTube. C'est à qui privatisera le plus vite le Web !

\section{En conclusion}

Quelles sont les conditions du décollage de l'Afrique dans la révolution numérique, alors que l'Afrique regorge de ressources naturelles? Certains posent l'urgence de corriger les handicaps suivants ${ }^{34}$ :

- la faillite des politiques des pays qui, sitôt sortis du système colonial, n'ont fait que perpétuer les pratiques de leurs anciens maîtres,

- la corruption endémique de l'appareil administratif, toléré par une oligarchie encore plus corrompue et qui encourage le clientélisme,

- la transformation de l'agriculture en monoculture. Les cultures villageoises qui, en plus de nourrir la population, pourraient être un puissant levier pour une politique industrielle,

- l'inadéquation du système éducatif. Non seulement celui-ci ne réussit pas à former ses travailleurs, mais il ne peut retenir ses professionnels formés à l'étranger,

- l'instabilité politique, due à une oligarchie qui perpétue son contrôle de décennie en décennie, souvent en transformant ses mandats en une durée infinie et en créant des dictatures qui se prolongent de père en fils.

\section{Les facteurs positifs}

- le rôle de l'Afrique du Sud, pays en émergence, dont l'abolition de l'apartheid n'a pas eu les effets désastreux promis par les prophètes de malheur et qui peut devenir un moteur pour le développement de l'Afrique,

- l'augmentation de la croissance du PIB, parfois en dizaine de points, alors que les pays développés se contenteront d'un maigre $1 \%, 2 \%$ ou $3 \%$, encore pour quelques années ${ }^{35}$.

Le dynamisme de la jeunesse de sa population; $70 \%$ de la population a moins de 30 ans, ce qui a constitué un handicap jusqu'à présent, mais qui peut devenir un atout, alors que les pays développés connaissent un vieillissement accéléré, comme l'a fait l'Inde, la 
nécessité de se réapproprier son secteur agricole, source de richesse. Il est important de ne pas le sacrifier en le modernisant au profit du secteur des NTIC, une nouvelle tendance de se doter de gouvernements élus démocratiquement, comme en Tunisie, au Sénégal, au Burkina Faso, en République centrafricaine. D'autres viendront à adopter cette même rigueur démocratique, comme l'exige la jeunesse de ces pays, la nouvelle donne en Éthiopie qui commence à se doter d'un secteur manufacturier, conséquence de la délocalisation de l'industrie en Chine. La Chine par ailleurs commence à investir beaucoup dans plusieurs pays africains.

Ce ne sont que quelques indications sur les signes prometteurs d'une renaissance probable du continent. L'Afrique doit forger son propre modèle de développement en tenant compte de ses forces et faiblesses, au moment où l'ensemble du monde a intérêt à l'aider à régler les problèmes généraux de l'exode migratoire, de l'épidémie mortifère du terrorisme de l'État islamique (EI) et des changements climatiques.

\section{BIBLIOGRAPHIE}

Boni Teiga Marcus, «Pourquoi l'Afrique ne se développe pas », Slate Afrique, 2006 (mis à jour en 2011), disponible sur www.slateafrique.com.

Agbobli Christian et Aubin France, «Perspectives critiques sur la communication pour le développement ", Revue française des sciences de l'information et de la communication [En ligne], 4 | 2014, mis en ligne le 01 janvier 2014, consulté le 30 août 2016. URL : https:// journals.openedition.org/rfsic/915 ; DOI : 10.4000/rfsic.915.

Diangitukwa Fweley, « Il est temps de sortir l'Afrique de l'impasse », texte de la conférence donnée à l'occasion du 50e anniversaire de l'Union africaine, 2013, disponible sur fweley.wordpress.com/2013/10/16/il-est-temps-de-sortir-de-limpasse.

Ford Martin, The Lights In the Tunnel : Automation, Accelerating Technology and the Eco- nomy of the Future, Paperback @ Amazon.com, 2009.

Godbout Jacques T., L'Esprit du don, avec la collaboration de Caillé Alain, La Découverte, coll. « Poche », Paris, 2000 (1re éd. 1992).

Kabou Axelle, Et si l'Afrique refusait le développement, L'Harmattan, Paris, 1991.

Lafrance Jean-Paul, « De la nécessité d'un modèle africain des NTIC », dans Kiyindou Alain (dir.), Communication pour le développement. Analyse critique des dispositifs et pratiques pro-fessionnelles au Congo, Éditions modulaires européennes, coll. «Échanges », Cortil-Wodon, 2008, p. 139-156.

Lafrance Jean-Paul, « Revisiter le concept du sous-développement et les modèles de communication qui accompagnent l'aide au développement ", Les Cahiers du CEDIMES, vol. 5, nº 3 (Le Développement durable en manque de communication), 2011, p. 33-44, disponible sur http:// cedimes.com.

Lafrance Jean-Paul, Laulan Anne-Marie, Rico de Sotelo Carmen (dir.), Place et rôle de la communication dans le développement international, Presses de l'Université du Québec, coll. "Communication », Québec, 2006. 
Lafrance Jean-Paul, La civilisation du CLIC ; La vie moderne, sous l'emprise des médias et des nouvelles technologies, Paris, l'Harmattan, 2013.

Rist Gilbert, Le Développement. Histoire d'une croyance occidentale, Presses de la Fondation nationale des sciences politiques, coll. « Références inédites », Paris, 1996.

Rostow Walt Whitman, The Stages of Economic Growth : A Non-Communist Manifesto, Cambridge University Press, Cambridge, 1960.

\section{NOTES}

1. Rist, 1996.

2. Rist, 1996, p. 69.

3. Lafrance, Laulan, Rico de Sotelo (dir.), 2006.

4. Discours présidentiel du président Truman, point IV, traduction établie sur la base des Public Papers of the Presidents, 1949, p 114-115, cité par Gilbert Rist, p. 118.

5. Lafrance, 2011, p. 33-44.

6. Autrefois, on parlait de pays colonisateurs et de pays colonisés, ces derniers étant davantage considérés comme des comptoirs pour se procurer des matières premières et de la main- d'œuvre bon marché.

7. Rostow, 1960.

8. Souvent, on attribue les piètres résultats de l'apport d'énormes richesses à la «maladie dite hollandaise ", qui se définit comme le phénomène économique qui relie l'exploitation des ressources naturelles et le déclin de l'industrie manufacturière ou agricole locale, inspiré du cas des Pays-Bas des années 1960. Il faut dire que dans le cas des monarchies du Golfe persique, les élites locales se sont accaparées les rentes colossales du pétrole uniquement à leur avantage. Certains pays comme le Venezuela ou le Nigeria vivent la baisse actuelle des prix du pétrole comme une catastrophe, signe que les richesses de l'or noir n'ont en rien réglé la situation de leur pays.

9. http://www.lemonde.fr/festival/article/2015/05/29/penser-la-civilisation-numerique_ 4643627_4415198.html

10. Agbobli et Aubin, 2012.

11. ibidem.

12. À titre d'exemple, l'Union Européenne condamne Apple à rembourser 13 milliards d'euros au trésor irlandais à titre « d'avantages fiscaux indus ». En 2015, Apple aurait payé en impôt 0,004 \% et $0,01 \%$ l'année précédente. Rappelons que l'entreprise de Cupertino a une capitalisation boursière de 570 milliards d'euros et un chiffre d'affaires de 234 milliards d'euros en septembre 2015. Évidemment l'entreprise va faire appel de la décision, et ce qui plus étonnant, l'Irlande aussi ainsi que les États-Unis !

13. Kabou, 1991, p 21.

14. Godbout, 2000.

15. Voir le rapport du groupe Cyrano : Financement sur l'économie sociale au Québec: Estimation de la taille du marché et idées nouvelles (1911) par Campbell, Allaire, Normand et Yakonowski. Selon le plan gouvernemental en économie sociale 2015-2020 le Québec compte environ 7000 entreprises d'économie sociale. Elles procurent un emploi à plus de 150000 personnes, ce qui représente un peu moins de $4 \%$ de l'emploi total au Québec. Le nombre d'emplois au sein des entreprises d'économie sociale est comparable au niveau d'emploi observé dans des secteurs comme le commerce de gros ou les services aux entreprises. En 2002, le chiffre d'affaires annuel des entreprises d'économie sociale était de 17 milliards de dollars. Aujourd'hui, uniquement pour les entreprises constituées en coopérative ou en mutuelle, ce chiffre atteint 33,4 milliards de dollars. 
http://www.economie.gouv.qc.ca/fileadmin/contenu/publications/administratives/

plans_action/plan_action_economie_sociale_2015-2020.html.

16. Le portable révolutionne l'Afrique, le journal Le Monde: http://www.lemonde.fr/idees/ article/2013/08/18/le-portable-revolutionne-l-afrique_3463006_3232.html. Voir aussi l'article de la revue TIC \& Société, «Téléphone mobile, Internet et développement : l'Afrique dans la société de l'information? » de Gado ALZOUMA (https://ticetsociete. revues.org/488).

17. Lafrance, 2008.

18. Karl Rettino-Parazelli, Le Devoir, Montréal, 16 janvier 2016.

19. Conclusions de la Banque mondiale, citées dans Échec des thèses libérales, par Gérard Bérubé, Le Devoir, 3 mars 2016.

20. NBIC : Nanotechnologies, Biotechnologies, Intelligence artificielle et sciences Cognitives. Voir entre autres, Humanisme Numérique : Valeurs et modèles pour demain? 5e rencontre annuelle des membres du Réseau internatio- nal des chaires UNESCO en communication (ORBICOM) les 26, 27 et 28 octobre 2016, à Paris, France, organisée par la Chaire ITEN de l'université Paris 8.

21. Le raisonnement de John Mitchell est le suivant: "Á job égal, les salariés ne sont pas compétitifs avec les machines, d'abord parce qu'ils sont moins efficaces, ensuite parce qu'ils représentent de lourdes charges sociales pour l'employeur ». L'idée de la cotisation Rose est donc de rétablir l'équilibre pour qu'il n'y ait pas de « concurrence déloyale » accrue entre humains et machines, lesquelles doivent devenir les «nouvelles vaches à lait ", entretien de J. Mitchell, Libération, 12 juin 1998.

22. Cité par Francis Mizio, «L'homme qui voulait taxer les robots », Libération, 22 mai 1998.

23. Voir l'article de Samson Okalow, «La technologie vous simplifie la vie ; pourrait-elle bientôt vous voler votre emploi ?", Canadian Business, 3 mai 2013.

24. Les robots détruiraient trois millions d'emplois d'ici à 2025 en France, La Tribune. fr, 27 octobre 2014.

25. Ford, déjà cité, 2009.

26. Les regards se tournent vers la Finlande qui versera 800 euros/mois à tous ses citoyens; un salaire garanti pour tous, http://www.journaldemontreal.com/2015/12/13/un-salaire-de-basegaranti-pour-tous.

27. Camille Bas-Worhlert, "En Suède, travailler moins sans perte de salaire. Les expériences tentées montrent les limites que pose la mondialisation », Agence France- Presse, Le Devoir, 27 juillet 2016. «Ni le patronat, ni les syndicats - qui ne croient pas au partage du travail par la réduction du temps de travail - le poussent à la roue. Or ce sont eux qui font la pluie et le beau temps dans le champ social depuis les années 30 ».

28. Selon une récente étude du Centre pour l'innovation, la technologie et la stratégie numériques de l'école de gestion Robert $\mathrm{H}$. Smith de l'université du Maryland, Facebook aurait permis de créer entre 180 et 240000 emplois indirects aux États-Unis, grâce au développement de l'édition d'applications, rapporte L'Expansion, article de Hubert Guillaud: http:// www.internetactu.net/2012/02/01ou-va-leconomie-numerique-13-vers-une-innovation-sansemplois.

29. Andrew McAfee et Erik Brynjolfsson, Race Against The Machine, Kindle edition, Digital Frontier Press, 2011. Selon un scénario jugé «tout à fait probable" par une étude de Roland Berger commandée par le JDD (Journal du Dimanche) et publiée le 26 octobre 2015, les robots seraient responsables de la suppression de 3 millions d'emplois en France, d'ici à 2025 en raison de 20 \% des tâches devenues automatisées.

30. Lafrance, 2013, p. 155 et suivantes.

31. Cette économie du partage pourrait représenter 235 milliards de dollars à l'échelle mondiale d'ici 2025, contre seulement 15 milliards fin 2014, selon des estimations du cabinet PriceWaterhouseCooper ( $\mathrm{PwC}$ ), publié par l'Express/Expansion, le 20 décembre 2015. 
32. Définition de Wikipédia: Les communs sont des ressources culturelles et naturelles accessibles à tous les membres d'une société. Ils incluent des éléments naturels dont l'air, l'eau, les sols et une terre habitable, mais il peut aussi s'agir de ressources culturelles immatérielles (Wikipédia est un exemple de commun).

33. Tim Berners-Lee, le créateur du WWW déclarait dans une entrevue à Radio-Canada le 23 août 2016: «un aspect important de la philosophie première du Web était la décentralisation. Maintenant, chaque entreprise essaie de se constituer un petit jardin intérieur qui ne peut jamais se comparer à la jungle folle qui pousse en toute liberté à l'extérieur. Vous devez prendre soin du Web, pensez à le protéger ».

34. Boni Teiga, 2006.

35. La croissance économique en Afrique sub-saharienne, passant de 4,7\% en 2013 à 5,2\% en 2014 selon les chiffres de la Banque mondiale (http://www.banquemondiale.org), devrait continuer d'augmenter. Selon Makhtar Diop, un grand nombre de pays d'Afrique sont régulièrement, ces dernières années, en tête du palmarès des pays à la croissance la plus rapide, $\mathrm{du}$ fait de l'adoption de réformes macroéconomiques pertinentes et de la prise de conscience du reste du monde du potentiel du continent aussi bien au niveau des échanges, des investissements et des affaires que de la science, de la technologie et du tourisme.

\section{RÉSUMÉS}

Gilbert Rist considérait le « développement » comme une utopie occidentale et un élément de la religion moderne du progrès des civilisations. Ce concept peut-il s'appliquer comme tel en Afrique? L'auteur analyse la naissance politique du paradigme, dit développementiste, en le situant à la fin de la Deuxième Guerre mondiale, au moment où la politique extérieure des ÉtatsUnis est en pleine redéfinition.

Mais qui dit développement, dit sous-développement, ou pour être plus poli de pays en voie de développement, avec tout ce que cela implique de biais pour ceux-ci. Quoiqu'il en soit, l'Afrique peut-elle se permettre de bouder le développement technologique ? La grande question est de se demander de quel type de développement l'Afrique a besoin? Revue des aspects positifs et des aspects négatifs du type de développement des pays africains et les chances de réussite.

Gilbert Rist saw “development” as a Western utopia and as an element of the civilizations' modern religion of progress. Can this concept be transposed to Africa? The author analyses the political birth of this paradigm, called developmentalism, taking place in the final stages of World War 2, when the foreign policy of the United States is undergoing fundamental changes. However, development often meansunderdevelopment, or developing countries to be polite, and all its implied biases. Nonetheless, can Can Africa afford to ignore development in its technological aspects? The real question is what type of development Africa truly needs? Review of the positive and negatives aspects of the development model adopted by African countries and its chances of success. 
INDEX

Mots-clés : idéologie, développement, Afrique

Keywords : ideology, development, Africa

\section{AUTEUR}

\section{JEAN-PAUL LAFRANCE}

Fondateur du secteur académique des communications à l'Université du Québec à Montréal (UQAM)

Chercheur invité au laboratoire ITEN de l'Université Paris 8 